\title{
A Computation Analysis on Flow Distribution in Microchannel under Triangular Manifold Arrangement System
}

\author{
Mahender Singh and Harpreet Singh* \\ Department of Mechanical Engineering, Chandigarh University, Gharuan - 140413, Punjab, India; \\ 16mme1020@gmail.com,erharpreets@gmail.com
}

\begin{abstract}
Background/Objectives: In this paper, a complete study and CFD analysis is performed on triangular manifolds and heat sink assembly with the help of different input parameters. The objective of the study is to get exact understanding of the temperature variation, pressure drop, and velocity variations at inlet and outlet of manifold when we pass fluid from one manifold to another by giving different heat inputs to the heat sink. CFD analysis for the simulation purpose is performed on the geometry for the evaluation of heat transfer coefficient, Nusselt number and Reynolds number. Methods/Statistical Analysis: In this study, for simulation CFD analysis of the geometry with different input parameters (velocity, density, viscocity specific heat, thermal conductivity, heat flux) is a simple method applied for the for different temperature and pressure drop values at inlet and outlet. Three arrangements for the water flow (U, S, and P) are designed in the manifolds through the heat sink. Four heat input values (5.06W, 7.53W.10.12W, 12.65W) are selected for the simulation. Findings: CFD investigation was performed with microchannel setup having 20 rectangular channels of $0.0005 \mathrm{~m}$ width, $0.0025 \mathrm{~m}$ depth and $0.2 \mathrm{~m}$ length with two triangular manifolds. Five input parameters (velocity, density, viscosity. specific heat, and thermal conductivity) and 24 numbers of experiments were performed with one temperature value at inlet $\left(25^{\circ} \mathrm{C}\right)$ for the comparison of temperature and pressure drop values. After the analysis, observation was found that temperature had different values at outlet of manifold in each fluid flow arrangement (U, S and P type) the Reynolds number range is varying from 599 to 899.The results show that the fluid flow was better in $U$ type fluid flow arrangement and the value of heat transfer coefficient $(96.57 \mathrm{w} / \mathrm{m} 2 \mathrm{c}$ to $161.52 \mathrm{w} / \mathrm{m} 2 \mathrm{c})$ is highest $(2-3 \%)$ in comparison to $S$ and P Type simulation. Applications: Triangular Manifolds are designed for providing uniform fluid flow and efficient heat transfer rate through the microchannel heat sink. As according to observation observed in the study, triangular manifolds have better heat transfer rate and it can be integrated in the next generation high temperature reactors. The manifolds are capable for use in fuel cells, liquid rocket engines, commercial heating and cooling and in automobiles.
\end{abstract}

Keywords: Heat Transfer Rate, Microchannel Heat Sink, Nusselt Number, Reynolds Number, Triangular Manifolds

\section{Introduction}

In the most of the industries when the flow of heat is required or we can say if we have to supply the fluid through the channels in the large quantity, the first important thing that comes in the mind of every engineer is, what type of manifold design we have and how the micro channels in the sink are arranged. We can enhance the heat flow rate by using the micro channels or in simple way if the different values of convective heat transfer rate are required, then there we use micro channels. In this study the $3 \mathrm{D}$ design is prepared for exact distribution and for proper evaluation of the pressure variations in the micro channels. For the results validation CFD analysis is performed. The main purpose of the study is to get the efficient mass flow rate at the outlet of the manifold. It is necessary to find the pressure drops, temperature difference and the flow distribution characteristics through the each channel because they affect the overall systems efficiency and performance. The triangular manifolds

${ }^{*}$ Author for correspondence 
are used and the flow is arranged in the three different ways $\mathrm{P}$ type fluid flow arrangement, $\mathrm{S}$ type fluid flow arrangement, $\mathrm{U}$ type fluid flow arrangement. The various parameters used for the simulation calculation are, viscosity, specific heat, density, thermal conductivity of the water, mass flow rate, heat flux, and the flow discharge.

The Reynolds and the Nusselt number are found for the comparison of the result after the calculation. On the outer two sides of the microchannel heat sink, manifolds are located which is used for the purpose that they have to pass the water in between them and manifolds are capable to keep the microchannel heat sink in the proper or in exact position while performing the experiment.

The model for the simulation is designed in such a way that, we can pass the proper or the exact supply of the heat to each channels surface. When the heat is supplied according to requirement for that purpose for the efficient heat transfer a heater is located below the channels and in the direction upward to the heat sink for the equal heat transfer rate on every channel. Material selected in this study for the model is aluminum and various tests are run on this material.

The total pressure drop after the simulation observed is maximum in the $\mathrm{u}$ type simulation and minimum in $\mathrm{p}$ type simulation. The main criteria for the evaluation of the micro channels performance are to create effective path for the fluid in manifold and in heat sink. Effective Channels arrangement in heat sink is a great source for the achievement of sufficient performance from the microchannels. The CFD simulation on the geometry is performed in three types. Entrance and exhaust for the water is designed in such a way that there are three water flow segments in the geometry. Three paths for the fluid flow Is U type fluid flow arrangement, $\mathrm{P}$ type fluid flow arrangement, $S$ type fluid flow arrangement. The various inputs for the test run for each fluid flow segment are velocity, thermal conductivity, viscosity, heat flux, density. After the calculation it has been found that in the U type arrangement the heat transfer coefficient is higher and in $\mathrm{p}$ type flow arrangement the pressure drop is minimum. In the simulation study different momentum equation and formulas are applied for the evaluation of the different parameters of the heat transfer. According to the statement of few authors, pressure drop or the heat transfer rate is the main concern in the study of microchannels and manifolds. In the study we performed all the tests on the triangular manifold but throughout the world the types of the manifold used by the various thermal engi- neering purposes or by the different researchers, are of different types. We can categorize the manifolds in the following different types, rectangular, convergent-divergent, semi-circular, triangular and trapezoidal manifolds. The fluid travel from the one manifold to another manifold through the micro channels in every case of the different type of the manifold. If the heat will not remove properly, the working temperature of the work piece may cross the desired temperature level, which then causes more chances for the failure rate of the equipment. So for the sufficient cooling conditions during the working in the electronic equipment micro channels were invented.

\section{Literature Survey}

In 1 investigate the heat transfer characteristics through a rectangular microchannel heat sink. Investigation is carried out experimentally and theoretically for the straight microchannel heat sink, water as the working fluid. From there observation they both stated that the temperature of the microchannel in the heat sink is increases with increase in the length of the channels. Observation shows that the heat sinks dissipate large amount of heat with relatively small surface temperature rise ${ }^{2}$. Indicate that simple air cooling in the microchannel is not sufficient for the high heat transfer purposes. They prefer the single phase cooling of the liquid as one of the best promising option for the high heat transfer rate purposes. Their study investigates the distribution in the microchannels. Experiment was performed on the two types of the manifolds. (Rectangular and trapezoidal manifold).After the investigation their observation stated that flow distribution is less in the microchannel with trapezoidal header as compared to the microchannel setup with the rectangular manifolds. The experiment also indicates that when rate flow reduces then mal distribution increases ${ }^{3}$ presents the study of the computational fluid dynamic model of the three types of manifolds rectangular, rectangular with semi-circular and divergent convergent. The 3D Simulation of every manifold is done in three ways (P type U type and in $\mathrm{S}$ type) with the three heat inputs (125W, 225W, 375W). From the study they describe that the heat transfer coefficient is more in the divergent convergent type manifold as compare to others in each case of heat input. Their study also justify that the Nusselt number more in the divergent convergent manifold than that of rectangular and the rectangular with semi-circular manifold ${ }^{4}$ indicates the Effects of manifold geometries on 
flow distribution to parallel microchannels. Investigation was performed on dimensionless geometric parameter indicating the optimal manifold configuration is derived through a numerical parametric study and validated by micro particle image velocimetry. By the validation methodology their observation shows different effects on the fluid flow distribution in the parallel microchannel heat sink 5 investigate the study of the fluid flow in the micro channels with Trapezoidal manifold for the misdistribution in the fluid configuration. They use of the 25 channels in the micro channels heat sink and two flow rates for the input supply and after the investigation they suggested that more mal distribution is achieved by the inline fluid flow configuration as compared to perpendicular inlet flow configuration and with the increment of the flow rate the flow misdistribution also decreases ${ }^{\underline{6}}$ investigate the study of the hydraulic parameters of the manifold for the achievement of the same mass flow rate at the outlet of the manifold. The experimentally 3D simulation was performed on the circular, tapered and on the longitudinal type manifolds. And after the simulation they suggested, as compared to the circular cross sectional manifolds the taper cross sectional manifold achieve uniform flow of the fluid $\mathrm{d}^{\mathrm{z}}$ performed numerical investigation for single phase flow pressure drop and heat transfer in rectangular metallic microchannels. Simulations were performed to investigate single phase flow and to evaluate heat transfer in copper rectangular microchannels.

Simulated were performed on two different configuration (a) single channel with hydraulic diameter of $0.561 \mathrm{~mm}$ and (b) multichannel configuration consisting of inlet and outlet manifolds and 25 channels with hydraulic diameter of $0.409 \mathrm{~mm}$. In ${ }^{8}$ performed the numerical study of the effect of the area of the manifold and the inlet and outlet flow arrangement on the flow distribution in the parallel rectangular microchannel cooling system.

They performed the simulation study on the twentyfive parallel micro channels with rectangular inlet and outlet manifold was simulated in three dimensional models. Flow distributions of three different flow arrangement $\mathrm{U}, \mathrm{Z}$ and I shapes are discussed in this section. The influences of inlet/outlet manifold area and inlet/outlet arrangement on flow distribution in channels were carried out. They stated that the large area of the inlet and the outlet manifold gives the low pressure drop. Moreover, a better uniformity of velocity and temperature distributions was achieved in I-shape flow arrangement. $\mathrm{In}^{9}$ performed the simulation study on three dimensional heat transfer analysis of microchannel heat sink by using Nano fluid. In the study the heat transfer in silicon based microchannel heat sink has been conducted using Nano fluid (TiO2-H2O) by simple method. Model of microchannel consists of trapezoidal channel. From the study they investigate that the heat transfer coefficient is high in comparison to the water as a coolant in micro channel heat $\sin ^{10}$ investigate the testing of the enhancement of the heat transfer in the rectangular micro channels. In their study they performed the numerical analysis for rectangular micro channels with sets of rectangular configurations, in order to find optimum configuration of micro channel. The study is performed for the three main purposes. 1. for design and the manufacturing of the rectangular microchannel, 2. development of the experimental setup for the rectangular microchannel, and 3. performance analysis of the rectangular microchannel. In ${ }^{11}$ investigate the convective heat transfers in a composite trapezoidal microchannel during magnetic heating. The analysis of conjugate heat transfer in a composite channel of trapezoidal cross-section is presented. The material for the channels was gadolinium in the study. Magnetic field is considered as the source of the heat generation in the study. The study mainly represent a systematic analysis of fluid flow and heat transfer processes during the magnetic heating of a magneto caloric material which is bonded to a silicon wafer. The simulation of conjugate heat transfer is a composite microchannel during magnetic heating was performed by varying the channel aspect ratio, Reynolds number, magnetic field strength and spacing between channels. The study investigated at a smaller flow rate, the outlet temperature increases as lower velocity fluid remains in contact with the solid for a longer period of time. According to the study if the fluid has the high velocity Nusselt number will increase with the decrease of the hydraulic diameter. Their study stated that water will give the best thermal performance with the higher values of the Nusselt number as compared to helium and FC-72.

\subsection{Microchannel Heat Sink}

The first major component in the geometry is microchannels. When heat is supplied to the microchannel heat sink by the heater, then the amount heat supplied dissipate large amount of heat flux in the geometry. For removal of high heat flux we use microchannels. If the microchannels were not taken into the consideration, it would never be possible to maintain an innovative and effective heat 
transfer rate. Microchannel heat sink with 20 microchannels setup is used in the study shown in Figure 1.

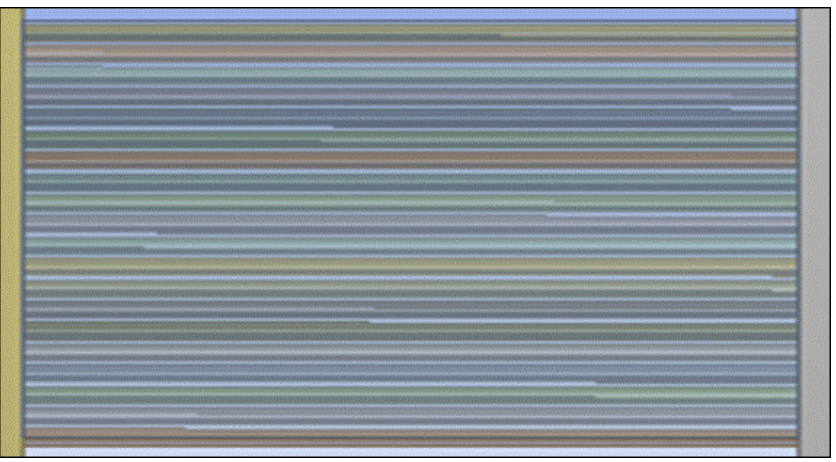

Figure 1. Micro channel heat sink.

\subsection{Manifolds}

Manifolds used in the study are basically a medium for the fluid circulation in the whole assembly in which the water through the porous side walls enters or leaves by the different range of the pressure. In the study the manifold used is triangular as shown in the figure. But as if we to categorize the manifolds, rectangular, divergent convergent and rectangular with semicircular is the most used manifolds by the engineers in the previous many decades as shown in the Figure 2.

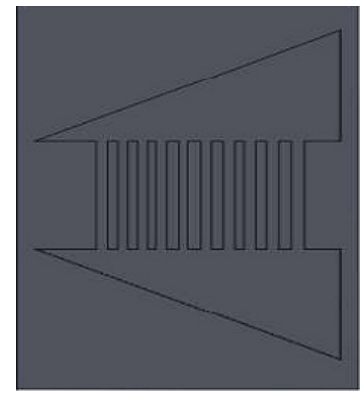

a) Triangle manifold
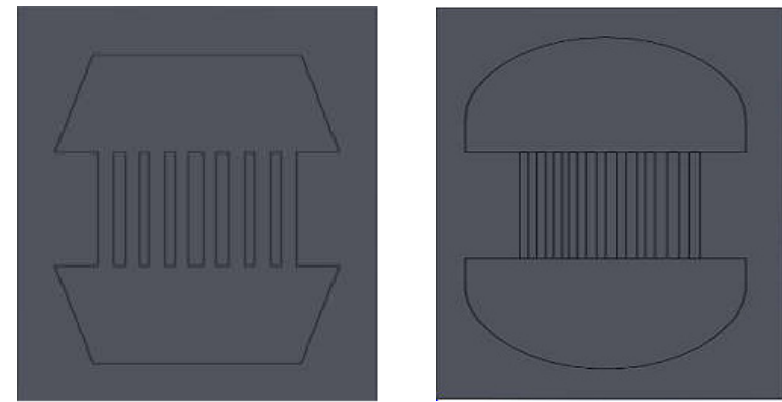

a) Divergent and convergent b) Rectangle with semi-circular Figure 2. Different types of manifolds.

\subsection{Electric Heater}

A rectangular shape section on the bottom side of the geometry was cut for the supply of the required heat fluxes during the simulation. To provide a good and effective heat flux as per according to the demand for the temperate variation, heater is used.

\section{Methodology}

The geometry of the assembly is shown in the Figure $3 \& 4$. The microchannel heat sink is arranged in between the two manifolds outside and one heater below the channels. The material selected for the manifolds in this study is aluminum. Different parameter taken while constructing the geometry for the simulation in the $3 \mathrm{D}$ software is shown in Table 1.

Table 1. Micro channels specification

\begin{tabular}{ll}
\hline Length of the channels & $2000 \mu \mathrm{m}$ \\
Width of the channels & $500 \mu \mathrm{m}$ \\
Depth of the channels & $2500 \mu \mathrm{m}$ \\
Spacing between the channels & $500 \mu \mathrm{m}$ \\
\hline
\end{tabular}

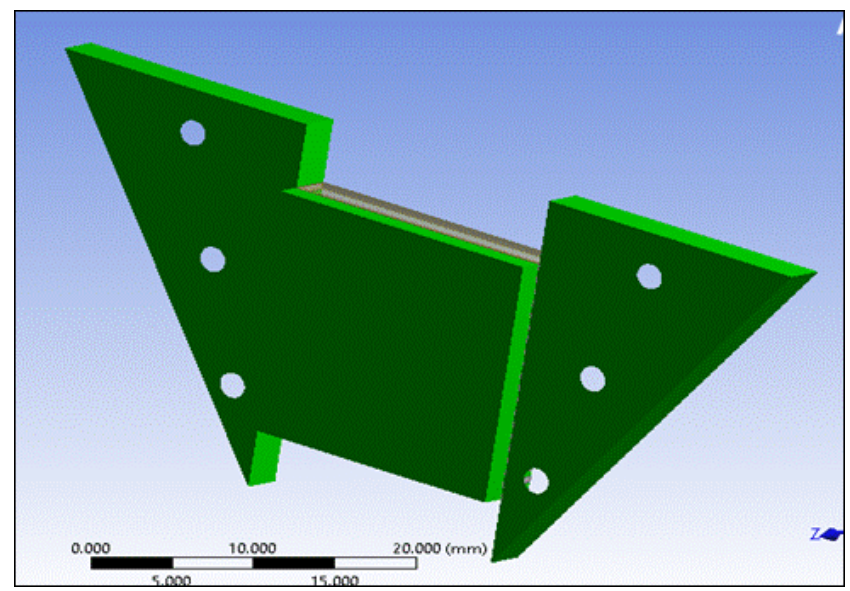

Figure 3. Design assembly of micro channels heat sink and manifolds.

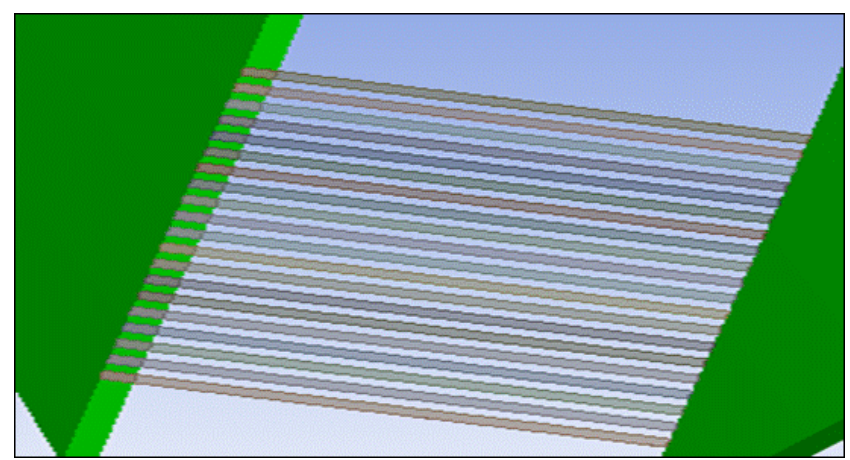

Figure 4. Micro channel. 
The width of the micro channels in the heat sink is $500 \mu \mathrm{m}$, the length of the channel is $20000 \mu \mathrm{m}$, Channels depth was considered $2500 \mu \mathrm{m}$ and spacing between channels was kept $500 \mu \mathrm{m}$. Heat sink is kept in center, surrounded with the two manifolds having 20 microchannels in it. For the different test run the fluid taken for the simulation is water.

During the geometry construction in the 3D software of simulation, the microchannel specification is not only the one requirement for the construction; there we had some hydraulic parameters which were taken in consideration for the geometry construction. The detail of the hydraulic parameters taken is shown in Table 2. Three cases (U, P S) in the geometry for the water flow are designed as mentioned in the previous article. For each case there is eight run of the simulation. For the first four run the value of the velocity is considered 0.030864 and the heat inputs for the first, second, third and fourth flow is $5.06 \mathrm{w}, 7.53 \mathrm{w}, 10.12 \mathrm{w}$. and $12.65 \mathrm{w}$ respectively and for the further four run the value of velocity is considered 0.046296 and the heat inputs values is again same $5.06 \mathrm{w}, 7.53 \mathrm{w}, 10.12 \mathrm{w}$ and $12.65 \mathrm{w}$. Viscosity, density, specific heat and thermal conductivity values are kept same for the each run. The simulation is performed 24 times on the geometry. For the further simulation calculation the final values after the results is calculated.

Table 2. Hydraulic parameters taken

\begin{tabular}{rll}
\hline Sl. No. & \multicolumn{1}{c}{ Parameters } & \multicolumn{1}{c}{ Values } \\
\hline 1. & Velocity of water $(\mathrm{m} / \mathrm{s})$ & $0.030684,0.046296$ \\
2. & Density of water $\left(\mathrm{kg} / \mathrm{m}^{\wedge} 3\right)$ & 998.1998 \\
3. & Specific heat $(\mathrm{j} / \mathrm{kgc})$ & 4181.4 \\
4. & Viscosity $(\mathrm{kg} / \mathrm{ms})$ & 0.001002 \\
5. & Thermal conductivity $(\mathrm{w} / \mathrm{mc})$ & 0.59 \\
6. & Design of the manifold & Triangular \\
7. & Reynolds number & $599-899$ \\
8. & Heat flux $(\mathrm{w})$ & $5.06,7.53,10.12,12.65$ \\
9. & Hydraulic diameter taken $(\mathrm{h})$ & 0.019512 \\
10. & Number of experiment & 24 \\
\hline
\end{tabular}

Formulae are applied for the calculation:

1. Reynolds Number

$$
\operatorname{Re}=\frac{\operatorname{Pvdh}}{\mu}
$$

2. Average Temperature

$$
\text { Tavg }=\frac{\mathrm{T}_{1}+\mathrm{T}_{2}}{2}
$$

3. Temperature Difference

$$
\Delta \mathrm{T}=\text { Twall-Tavg }
$$

4. Flow Discharge

$$
\mathrm{Q}=\mathrm{m} \mathrm{Cp} \Delta \mathrm{t}
$$

5. Heat Transfer Co efficient

$$
\mathrm{h}=\frac{\mathrm{q}}{\mathrm{As} \Delta \mathrm{t}}
$$

6. Nusselt Number

$$
\mathrm{Nu}=\mathrm{h} \frac{\mathrm{Dh}}{\mathrm{k}}
$$

\section{Results and Discussion}

In the experiment study the simulation has been worked at two different flow rates and at four different heat inputs $(5.06 \mathrm{w}, 7.53 \mathrm{w}, 10.12 \mathrm{w}$ and $12.65 \mathrm{w})$. The final values show that the manifold has the best results in u type fluid flow arrangement comparatively to $\mathrm{P}$ and $\mathrm{S}$ type. In the $\mathrm{u}$ type flow arrangement pressure drop is minimum and the heat transfer coefficient is higher in this study of $U$ type fluid flow as compared to P and S. In the study there is eight simulations run in each arrangement. In $U$ type fluid flow arrangement the heat transfer coefficient value is $105.0217 \mathrm{w} / \mathrm{m} 2 \mathrm{c}$ to $99 \mathrm{w} / \mathrm{m} 2 \mathrm{c}$ in case of first four run and the value of heat transfer coefficient for further four simulation run ranges from $157.57 \mathrm{w} / \mathrm{m} 2 \mathrm{c}$ to $144.36 \mathrm{w} /$ $\mathrm{m} 2 \mathrm{c}$. The Nusselt number is little higher in $\mathrm{P}$ type fluid flow arrangement than that of $S$ and $U$ type. The range of Nusselt number in each case is almost 3 to 5 .

\section{Conclusion}

Probably from the result we find there are small variation in the heat transfer coefficient and the pressure drop in each of the simulation. From the observation it was observed that wall temperature of manifolds and the microchannel heat sink always lies in between the inlet and outlet temperature of the manifolds. In all three simulation $(\mathrm{S}, \mathrm{P}, \mathrm{U}$ 
TYPE) the design model is safe, but as if we have to determine the best fluid arrangement from these three cases on the basis of heat transfer coefficient and pressure drop than the $\mathrm{U}$ type simulation is better than that of $\mathrm{P}$ and $\mathrm{S}$ type because the pressure drop in the $\mathrm{U}$ type fluid flow is minimum as compare to others and the heat transfer coefficient in $\mathrm{U}$ type simulation run is higher than the $\mathrm{S}$ an $\mathrm{P}$ type simulation. The value of the Heat transfer coefficient increases in $\mathrm{U}$ type simulation than that of the P type simulation by $1.02 \%$ at $5.09 \mathrm{w}, 2.11 \%$ at $7.59 \mathrm{w}, 0.44 \%$ at $10.12 \mathrm{w}$ and $0.34 \%$ at $12.59 \mathrm{w}$. The value of heat transfer coefficient increases in $\mathrm{U}$ type simulation than that of the $\mathrm{S}$ type fluid flow arrangement by $0.10 \%$ at $5.09 w, 3.68 \%$ at $7.59 w, 3.43 \%$ at $10.12 \mathrm{w}$ and $0.30 \%$ at $12.63 \mathrm{w}$. And the Reynolds number variation occurs from 599 to 899 .

\section{References}

1. Gawali BS, Swami VB, Thakre SD. Theoretical and experimental investigation of the heat transfer characteristics through a rectangular microchannel heat sink, Journal of the Innovative Research in the Science, Engineering and Technology. 2014; 3(8):1-10.

2. Anbumeenakshi C, Thansekhar MR. Experimental Investigation of Flow maldistribution microchannels, Journal of Innovative Research in Science, Engineering and Technology. 2014; 3(3):1-4.

3. Anurag Dahiya, Satbir Singh Sehgal, Harpreet Singh. CFD and heat transfer analysis of microchannel heat sink with different inlet and outlet flow arrangements, Indian Journal of Science and Technology. 2016; 9(36):1-5. https://doi. org/10.17485/ijst/2016/v9i36/101406.

4. Kim D, Yu C, Yoon SH, Choi S. Effects of manifold geometries on flow distribution to parallel microchannels, Journal of Mechanical Science and Technology. 2011; 25(12):3069-74. https://doi.org/10.1007/s12206-011-1220-3.
5. Anbumeenakshi C, Thansekhar MR, Srinivasan M, Venkatesan M. Effect of flow inlet on flow distribution in microchannel heat sink, Journal of Chemical and Pharmaceutical Sciences. 2015; 1-3.

6. Hassan JM, Mohamed TA, Mohammed WS, Alawee WH. Modelling the Uniformity of Manifold with Various Configurations, Journal of Fluids. 2014; 1-8.

7. Sahar AM, Özdemir MR, Fayyadh EM, Wissink J, Mohamed M. Single phase flow pressure drop and heat transfer in rectangular metallic microchannels, Journal of Applied Thermal Engineering. 2016; 93:1324-36.

8. Sahara AM, Shaifulc AIM. Numerical study of the effect of the area of the manifold and inlet/outlet flow arrangement on flow distribution on parallel rectangular microchannel cooling system, Journal Teknologi (Sciences and Engineering). 2017; 7(3):81-87.

9. Agnihotri A, Sharma A. Three dimensional heat transfer analysis of microchannel heat sink using nano fluid, International Journal of Science, Technology and Management. 2015; 4(1)1-10.

10. Rohit Pingale, Siddaraj Allurkar, Girish Gudi. Testing of heat transfer enhancement in rectangular microchannels, International Journal of Advance Engineering and Research Development. 2016; 3(12):1-8.

11. Rahman MM, Shevade SS, Ojada E. Convective heat transfer in a composite trapezoidal microchannel during magnetic heating, International Communications in Heat and Mass Transfer. 2010; 37:1175-81. https://doi. org/10.1016/j.icheatmasstransfer.2010.07.017.

12. Kumaraguruparan G, Manikandakumaran R, Sornakumar T. Numerical and the experimental investigation of the flow maldistribution in a microchannel heat sink, Journal of the Heat and Mass Transfer. 2011; 38:1349-53. 